\title{
SHARP INEQUALITIES FOR POSITIVE FUNCTIONS
}

\author{
JOHNNY E. BROWN
}

This paper is dedicated to Peter L. Duren on the occasion of his 70th birthday.

\begin{abstract}
In this paper we determine the extreme points for the families of positive and positive real functions. We prove a number of new sharp inequalities in addition to quantifying and generalizing some well-known results. We also investigate sharp bounds on $\left|p^{\prime}(z)\right|$ and $\left|p^{\prime \prime}(z)\right|$ for positive real functions, unknown since 1932 .
\end{abstract}

\section{INTRODUCTION}

Let $H^{+}$denote the open right half-plane. The collection of all functions $p(z)$ analytic in $H^{+}$and satisfying $\Re e p(z)>0$ for $z \in H^{+}$is called the class of positive functions and is denoted by $P$. The class of positive functions which satisfy $p(\bar{z})=\overline{p(z)}$ is called the class of positive real functions, denoted by $P R$. Apart from their mathematical interest, positive real functions arise as the complex impedance of passive two-terminal networks where $z$ is the complex frequency (see [4] or [1]). In particular, it was shown by O. Brune [4] in 1931 that each such system gives rise to a positive real function and conversely.

The complex impedance in an alternating current may be written in the form $R+i X$, where $R \geq 0$ is the resistance and $X$ is the reactance. Materials with low, medium and high resistances are conductors, semiconductors and dielectrics, respectively. Reactance appears when there is opposition to the flow of current. When an alternating current passes through a circuit containing reactance, two possibilities may occur: energy may be stored and released as a magnetic field and hence $X>0$ (inductive), or energy may be stored and released in the form of an electric field and hence $X<0$ (capacitive).

The purpose of the present paper is to give a new and unified approach to estimates and properties of positive and positive real functions. We present alternate proofs of some known results and also give some new quantitative estimates for these results. All the estimates obtained are sharp. The problem of determining the sharp upper bounds for $\left|p^{(n)}(z)\right|$ for positive real functions has been open since 1932 (see [16] and [7]). We show how to determine this for all $n \geq 1$. The particular cases $n=1$ and $n=2$ are considered. Sharp growth estimates on the absolute value of the reactance term, $\Im m p(z)$, for positive real functions are also given for the first time.

2000 Mathematics Subject Classification. Primary 30D50; Secondary 30 D99.

Key words and phrases. Positive functions, functions of positive real part, extreme points. 
For comparison purposes we summarize some of the principal known results for $P$ and $P R$, all proved by a variety of methods:

(A) If $p \in P$, then $\lim _{|z| \rightarrow \infty} p^{\prime}(z)=\lim _{|z| \rightarrow \infty} \frac{p(z)}{z}=\lim _{|z| \rightarrow \infty} \frac{\Re e p(z)}{\Re e z}=A \geq 0$, uniformly in the wedge $|\arg z| \leq \mu<\frac{\pi}{2}$. ([6], [9], [15], [16])

(B) If $p \in P$ is analytic at $z=i \beta(\beta \in \mathbb{R})$ and it is a zero of $p$, then it must be a simple zero and $p^{\prime}(i \beta)>0$. (Brune [4])

(C) If $p \in P$ has a pole at $z=i \beta(\beta \in \mathbb{R})$, then it must be a simple pole with positive residue. (Brune [4])

(D) If $p \in P$, then $\left|p^{(n)}(z)\right| \leq \frac{n ! \Re e p(z)}{(\Re e z)^{n}}, n=1,2,3, \ldots$ This is sharp for $z=x_{0}+i y_{0}$ for the function $p(z)=x_{0} /\left(z-i y_{0}\right)^{-1}$. (Wolff and DeKoK [16], Goldberg [7]; and for $n=1$, Reza[11])

(E) If $p \in P$ and $C$ is a Jordan $\operatorname{arc}$ in $|\arg z| \leq \mu<\frac{\pi}{2}$, which ends at $z=0$, and $\Re e p(z)$ is bounded on $C$, then $\Re e p(z)$ is uniformly bounded in the sector $|\arg z| \leq \phi,|z| \leq 1$ for any $0 \leq \phi<\frac{\pi}{2}$. (Tsuji [14])

(F) If $p \in P R$, then

$$
\begin{aligned}
& \text { (a) } \frac{\Re e z}{|z|^{2}+1}<\frac{\Re e p(z)}{p(1)} \leq \frac{|p(z)|}{p(1)}<\frac{|z|^{2}+1}{\Re e z} . \quad \text { (Richards [12]) } \\
& \text { (b) } \frac{1}{|z|(1+B(z))} \leq \frac{|p(z)|}{p(1)} \leq|z|(1+B(z)), \text { where } \\
& B(z)=\left\{\begin{array}{cc}
\left(1+(\Re e z)^{2}\right) /(\Re e z)^{2} & , \Im m\{z\}=0 \\
\left(1+|z|^{2}\right)\left|\arg \left(z^{2}\right)\right| /\left|\Im m\left\{z^{2}\right\}\right| & , \Im m\{z\} \neq 0
\end{array} .\right. \text { (Seshu and Seshu [13]) }
\end{aligned}
$$

(G) If $p \in P R$, then $|\operatorname{Arg} p(z)| \leq|\operatorname{Arg} z|$, where Arg denotes the principal value of the argument. (Brune [4])

\section{Positive Functions}

For each fixed $\omega_{0}=u_{0}+i v_{0}, u_{0}>0$, we define $P\left(\omega_{0}\right)$ as the subclass of functions $p \in P$ for which $p(1)=\omega_{0}$. Thus each $p \in P$ belongs to precisely one class $P\left(\omega_{0}\right)$. We can now state our main results on positive functions.

Theorem 2.1. Let $\omega_{0}=u_{0}+i v_{0} \in H^{+}$.

(a) Each $p \in P\left(\omega_{0}\right)$ has a representation

$$
p(z)=u_{0} \int_{T} \frac{(z+1)+x(z-1)}{(z+1)-x(z-1)} d \mu(x)+i v_{0},
$$

where $\mu(x)$ is a probability measure on the unit circle $T$.

(b) $P\left(\omega_{0}\right)$ is a compact convex family of functions. 
(c) The set of extreme points of $P\left(\omega_{0}\right)$ is given by

$$
\mathcal{E}\left(P\left(\omega_{0}\right)\right)=\left\{u_{0}\left[\frac{(z+1)+x(z-1)}{(z+1)-x(z-1)}\right]+i v_{0}:|x|=1\right\} .
$$

Proof: Define the linear fractional transformations $\psi$ and $\phi$ as follows

$$
\begin{gathered}
z=\psi(\zeta)=\frac{1+\zeta}{1-\zeta} \\
\zeta=\phi(z)=\frac{z-1}{z+1}
\end{gathered}
$$

If $p \in P\left(\omega_{0}\right)$, then the function

$$
q(\zeta)=\frac{p(\psi(\zeta))-i v_{0}}{u_{0}}
$$

is analytic in $|\zeta|<1$, 凡e $q(\zeta)>0$ and $q(0)=1$. Hence $q \in \mathbf{P}$, the well-known Carathéodory class, and so we obtain

$$
\frac{p(\psi(\zeta))-i v_{0}}{u_{0}}=\int_{T} \frac{1+x \zeta}{1-x \zeta} d \mu(x)
$$

where $\mu(x)$ is a probability measure on the unit circle $T$ (see [8], page 30). If we now let $\zeta=\phi(z)$ as in (2), then we obtain the representation (a).

From (a) we see that each $p \in P\left(\omega_{0}\right)$ is uniformly bounded on compact subsets of $H^{+}$and hence $P\left(\omega_{0}\right)$ forms a normal family. Compactness follows from the normalization $p(1)=\omega_{0}$. Additionally the class is convex since for any pair $p, q \in P\left(\omega_{0}\right)$, then $t p(z)+(1-t) q(z)$ clearly belongs to $P\left(\omega_{0}\right)$ for all $0 \leq t \leq 1$.

Finally, to prove (c), it suffices to show that the mapping $\mu \rightarrow p$ given in (a) is one-to-one (see [2], Theorem 1). For the Carathéodory class $\mathbf{P}$ it is known that the mapping $\mu \rightarrow q$ is one-to-one where

$$
q(\zeta)=\int_{T} \frac{1+x \zeta}{1-x \zeta} d \mu(x)
$$

Because the function $\zeta=\phi(z)$ given in (2.2) is univalent, the mapping $\mu \rightarrow p$ given in (a) is one-to-one.

The importance of identifying the extreme points is the key to our method because of the following results.

Lemma 2.2. [8] Let $\mathcal{F}$ be a compact, convex family of analytic functions and $\mathcal{E}(\mathcal{F})$ its set of extreme points. If $\Phi$ and $L$ are continuous real-valued convex and linear functionals, respectively, on $\mathcal{F}$, then

(a) $\max _{f \in \mathcal{F}} \Phi(f)=\max _{f \in \mathcal{E}(\mathcal{F})} \Phi(f)$

(b) $\max _{f \in \mathcal{F}} L(f)=\max _{f \in \mathcal{E}(\mathcal{F})} L(f)$ and $\min _{f \in \mathcal{F}} L(f)=\min _{f \in \mathcal{E}(\mathcal{F})} L(f)$ 
Another useful result for quotients of functionals is given by the following.

Lemma 2.3. [3] Let $\mathcal{F}$ be a compact, convex family of analytic functions. If $\Phi$ and $L$ are continuous real-valued convex and linear functionals, respectively, on $\mathcal{F}$ with $L(f)>0$ on $\mathcal{F}$, then $\max _{f \in \mathcal{F}} \frac{\Phi(f)}{L(f)}=\max _{f \in \mathcal{E}(\mathcal{F})} \frac{\Phi(f)}{L(f)}$.

We first prove a useful growth result for positive functions.

Theorem 2.4. If $p \in P\left(\omega_{0}\right)$, then

$$
u_{0}\left(\frac{|z+1|-|z-1|}{|z+1|+|z-1|}\right) \leq \Re e p(z) \leq\left|p(z)-i v_{0}\right| \leq u_{0}\left(\frac{|z+1|+|z-1|}{|z+1|-|z-1|}\right),
$$

for $\Re$ e $z>0$. This result is sharp.

Proof: It is well known that if $q \in \mathbf{P}$ then

$$
\frac{1-|\zeta|}{1+|\zeta|} \leq \Re e q(\zeta) \leq|q(\zeta)| \leq \frac{1+|\zeta|}{1-|\zeta|},|\zeta|<1
$$

If $p \in P\left(\omega_{0}\right)$, then this inequality applied to (2.3) gives

$$
\frac{1-|\zeta|}{1+|\zeta|} \leq \Re e\left\{\frac{p(\psi(\zeta))}{u_{0}}\right\} \leq\left|\frac{p(\psi(\zeta))-i v_{0}}{u_{0}}\right| \leq \frac{1+|\zeta|}{1-|\zeta|}
$$

Now let $\zeta=\phi(z)=\frac{z-1}{z+1}$ and the result follows.

To prove sharpness, fix $z_{0} \in H^{+}$and let $\frac{z_{0}-1}{z_{0}+1}=\rho_{0} e^{i \theta_{0}}$. The functions

$$
p_{1}(z)=u_{0}\left(\frac{(z+1)+e^{-i \theta_{0}}(z-1)}{(z+1)-e^{-i \theta_{0}}(z-1)}\right)+i v_{0}
$$

and

$$
p_{2}(z)=u_{0}\left(\frac{(z+1)-e^{-i \theta_{0}}(z-1)}{(z+1)+e^{-i \theta_{0}}(z-1)}\right)+i v_{0}
$$

give equality for the right and left-hand inequalities, respectively, at $z_{0}$.

We can now give an upper estimate for the constant $A$ in the limits given in (A). If we let $z=s>1$, then from Theorem 2.4 we obtain

Corollary 2.5. If $p \in P\left(\omega_{0}\right)$, then

$$
0<\frac{\Re e p(s)}{s} \leq u_{0}=\Re e \omega_{0}
$$

for all $0<s<\infty$. Equality holds for $p(z)=u_{0} z+i v_{0}$. 
From (A) we thus have the sharp estimate that

$$
A \leq u_{0}=\Re e p(1) .
$$

We should also point out that the constant $A$ can assume all values in $\left[0, u_{0}\right]$. Indeed, for any fixed $0 \leq \epsilon \leq 1$, the function $p(z)=(\epsilon z+1-\epsilon) u_{0}+i v_{0} \in P\left(\omega_{0}\right)$ and $\lim _{|z| \rightarrow \infty} p^{\prime}(z)=\epsilon u_{0}$.

The above estimate on $A$ appears to be new. There are many proofs of the limits in (A), but no estimates of the constant $A$. It is defined for example by Tsuji ([15], page 150) as

$$
A=\frac{1}{\pi} \lim _{m \rightarrow \infty} \lim _{n \rightarrow \infty} \int_{-\frac{\pi}{2}+\frac{L_{k}}{2 R_{n}}}^{\frac{\pi}{2}-\frac{L_{k}}{2 R_{n}}} \frac{\Re e p\left(R_{n}+R_{n} e^{i \phi}\right)}{2 R_{n} \cos ^{2} \phi} d \phi
$$

where $0<R_{1}<R_{2}<\cdots \rightarrow \infty$ and $0<L_{1}<L_{2}<\cdots \rightarrow \infty$. He also proved that

$$
\lim _{r \rightarrow \infty} \frac{1}{r} \int_{-\frac{\pi}{2}}^{\frac{\pi}{2}} \Re e\left\{p\left(r e^{i \theta}\right)\right\} \cos \theta d \theta=\frac{\pi}{2} A .
$$

However, it is difficult from these representations to see that $A \leq \Re e p(1)$.

The next result gives quantitative sharp estimates for the results $(B)$ and $(\mathrm{C})$.

Theorem 2.6. Let $p \in P\left(\omega_{0}\right)$ with $\omega_{0}=u_{0}+i v_{0} \in H^{+}$and $\beta \in \mathbb{R}$.

(a) If $z=i \beta$ is a zero of $p$, then it is simple and satisfies $\Re$ e $p^{\prime}(i \beta) \geq \frac{u_{0}}{\beta^{2}+1}$. This is sharp for all real $\omega_{0}$.

(b) If $z=i \beta$ is a pole of $p$, then it is simple with residue $|\operatorname{Res}\{p, i \beta\}| \leq u_{0}\left(\beta^{2}+1\right)$. This is sharp for all $\omega_{0} \in H^{+}$.

Proof: If $z=i \beta$ is a zero of $p \in P\left(\omega_{0}\right)$, then for some $\epsilon_{0}>0$, we have

$$
p(z)=\sum_{k=1}^{\infty} c_{k}(z-i \beta)^{k}
$$

valid for $|z-i \beta|<\epsilon_{0}$. Letting $z=\epsilon+i \beta, 0<\epsilon<\epsilon_{0}$ and using Theorem 2.4 we get

$$
\begin{aligned}
\Re e\left\{c_{1}\right\}+\Re e \sum_{k=2}^{\infty} c_{k} \epsilon^{(k-1)} & =\frac{\Re e p(z)}{\epsilon} \\
& \geq \frac{u_{0}}{\epsilon}\left(\frac{|z+1|-|z-1|}{|z+1|+|z-1|}\right) \\
& =\frac{u_{0}}{\epsilon}\left(\frac{|z+1|^{2}-|z-1|^{2}}{(|z+1|+|z-1|)^{2}}\right) \\
& =\frac{4 u_{0}}{\left(\sqrt{\beta^{2}+(1+\epsilon)^{2}}+\sqrt{\beta^{2}+(1-\epsilon)^{2}}\right)^{2}}
\end{aligned}
$$


Now let $\epsilon \rightarrow 0^{+}$to conclude that $\Re e\left\{c_{1}\right\} \geq \frac{u_{0}}{\beta^{2}+1}$. (Note that by (B) we know $p^{\prime}(i \beta)$ is real so in fact we have $c_{1}=p^{\prime}(i \beta) \geq u_{0}\left(\beta^{2}+1\right)^{-1}$.) For $\omega_{0}=u_{0}>0$, sharpness is achieved for $p(z)=u_{0}\left(\frac{z-i \beta}{1-i \beta z}\right)$. This proves $(\mathrm{a})$.

Now let us suppose $z=i \beta$ is a pole of $p \in P\left(\omega_{0}\right)$. Hence we have

$$
p(z)=\sum_{k=1}^{N} \frac{b_{k}}{(z-i \beta)^{k}}+g(z)
$$

where $g(z)$ is analytic in $|z-i \beta|<\epsilon_{0}$ for some $\epsilon_{0}>0$. Proceeding as above we let $z=\epsilon+i \beta, 0<\epsilon<\epsilon_{0}$, and use Theorem 2.4 to obtain

$\left|b_{1}+\sum_{k=2}^{N} \frac{b_{k}}{\epsilon^{k-1}}+\epsilon g(z)\right|=|\epsilon p(z)| \leq \epsilon u_{0} \frac{(|z+1|+|z-1|)^{2}}{|z+1|^{2}-|z-1|^{2}}+\epsilon\left|v_{0}\right| \leq u_{0}\left[\beta^{2}+(1+\epsilon)^{2}\right]+\epsilon\left|v_{0}\right|$.

If we let $\epsilon \rightarrow 0^{+}$, then we must have $b_{k}=0$ for $k=2,3, \cdots, N$ and $\left|b_{1}\right| \leq u_{0}\left(\beta^{2}+1\right)$. Sharpness occurs for all $\omega_{0} \in H^{+}$for $p(z)=u_{0}\left(\frac{1-i \beta z}{z-i \beta}\right)+i v_{0}$. (Since Brune [4] proved that $\operatorname{Res}\{p, i \beta\}>0$, we have $0<\operatorname{Res}\{p, i \beta\} \leq u_{0}\left(\beta^{2}+1\right)$.)

It is interesting to note that the same conclusion (a) holds in the more general setting where $\Re$ e $p(i \beta)=0$. In this case we have

$$
p(z)=i \gamma+\sum_{k=1}^{\infty} c_{k}(z-i \beta)^{k}
$$

where $\gamma \in \mathbb{R}$ and the proof proceeds exactly as above.

The next result shows that $\Re$ e $p(z)$ and $\Re e p(|z|)$ are comparable in sectors.

Theorem 2.7. If $p \in P\left(\omega_{0}\right)$, then

$$
\left(\frac{1-\left|\tan \frac{\theta}{2}\right|}{1+\left|\tan \frac{\theta}{2}\right|}\right) \leq \frac{\Re e p\left(r e^{i \theta}\right)}{\Re e p(r)} \leq\left(\frac{1+\left|\tan \frac{\theta}{2}\right|}{1-\left|\tan \frac{\theta}{2}\right|}\right)
$$

for $|\theta|<\frac{\pi}{2}$ and $r>0$. This result is sharp.

Proof: Observe first that if $q \in P(1)$ and $z=e^{i \theta}$, then Theorem 2.4 gives

$$
\left(\frac{1-\left|\tan \frac{\theta}{2}\right|}{1+\left|\tan \frac{\theta}{2}\right|}\right) \leq \Re e q\left(e^{i \theta}\right) \leq\left(\frac{1+\left|\tan \frac{\theta}{2}\right|}{1-\left|\tan \frac{\theta}{2}\right|}\right)
$$

Now fix $r>0$, let $p \in P\left(\omega_{0}\right)$ be arbitrary and then apply (2.6) to

$$
q(z)=\frac{p(r z)-i \Im m p(r)}{\Re e p(r)}
$$

to obtain the result. 
To demonstrate sharpness, assume without loss of generality $0 \leq \theta<\pi / 2$ and let

$$
q_{1}(z)=\left(\frac{(z+1)-i(z-1)}{(z+1)+i(z-1)}\right) \quad \text { and } \quad q_{2}(z)=\left(\frac{(z+1)+i(z-1)}{(z+1)-i(z-1)}\right) .
$$

For fixed $r>0$, the functions $p_{1}$ and $p_{2}$ give sharp upper and lower bounds for $z=r e^{i \theta}$, respectively, where

$$
p_{k}(z)=u_{0} \frac{q_{k}\left(\frac{z}{r}\right)}{\Re e q_{k}\left(\frac{1}{r}\right)}+i v_{0} .
$$

The above theorem allows us to quantify and generalize Tsuji's theorem (E) as well as give a different and more direct proof.

Corollary 2.8. Let $p \in P\left(\omega_{0}\right)$. If $m \leq \Re e p(z) \leq M$ on a Jordan arc $C$ lying in the sector $|\arg z| \leq \mu<\frac{\pi}{2},|z| \leq 1$, ending at $z=0$ and intersecting $|z|=1$, then 凡e $p(z)$ is uniformly bounded above and below in the sector $|\arg z| \leq \phi,|z| \leq 1$, for any $0 \leq \phi<\frac{\pi}{2}$. Moreover, for $z$ in that sector we have

$$
m\left(\frac{1-\tan \frac{\mu}{2}}{1+\tan \frac{\mu}{2}}\right)\left(\frac{1-\tan \frac{\phi}{2}}{1+\tan \frac{\phi}{2}}\right) \leq \Re e p(z) \leq M\left(\frac{1+\tan \frac{\mu}{2}}{1-\tan \frac{\mu}{2}}\right)\left(\frac{1+\tan \frac{\phi}{2}}{1-\tan \frac{\phi}{2}}\right) .
$$

Proof: For any fixed $z_{0}=r_{0} e^{i \theta_{0}}$ on $C,\left|\theta_{0}\right| \leq \mu<\frac{\pi}{2}$ and $r_{0} \leq 1$, from the inequality (2.5) we may deduce that

$$
\Re e p\left(r_{0}\right)\left(\frac{1-\tan \frac{\mu}{2}}{1+\tan \frac{\mu}{2}}\right) \leq \Re e p\left(r_{0} e^{i \theta_{0}}\right) \leq M .
$$

Thus we see that $\Re e p(r) \leq M\left(\frac{1+\tan \frac{\mu}{2}}{1-\tan \frac{\mu}{2}}\right)$, for all $r \leq 1$. If $|\theta| \leq \phi$ and $r \leq 1$, we apply inequality (2.5) again to obtain

$$
\Re e p\left(r e^{i \theta}\right) \leq\left(\frac{1+\tan \frac{\phi}{2}}{1-\tan \frac{\phi}{2}}\right) \Re e p(r) \leq M\left(\frac{1+\tan \frac{\phi}{2}}{1-\tan \frac{\phi}{2}}\right)\left(\frac{1+\tan \frac{\mu}{2}}{1-\tan \frac{\mu}{2}}\right) .
$$

A similar argument gives the lower bound.

It is of interest to note that Tsuji's Theorem [14] also contains the statement that if $\Re$ e $p(z) \rightarrow 0$ (or $+\infty$ ) as $z \rightarrow 0$ along $C$, then $\Re$ e $p(z) \rightarrow 0$ (or $+\infty$ ) uniformly when $z \rightarrow 0$ inside the sector $|\arg z| \leq \phi<\frac{\pi}{2},|z| \leq 1$. This follows immediately from our corollary.

Theorem 2.7 also gives a rather curious inequality related to Tsuji's result (2.4). For example it follows that if $p \in P\left(\omega_{0}\right)$, then

$$
\frac{\Re e p(r)}{r}(\pi-2) \leq \frac{1}{r} \int_{-\frac{\pi}{2}}^{\frac{\pi}{2}} \Re e\left\{p\left(r e^{i \theta}\right)\right\} \cos \theta d \theta \leq \frac{\Re e p(r)}{r}(\pi+2)
$$


for all $0<r<\infty$. While this does not prove (2.4), it gives bounds on these integrals which appear to be new. Note that if $p \in P\left(\omega_{0}\right)$, then $f(\theta)=\Re e p\left(r e^{i \theta}\right) \cos \theta \in$ $L^{1}\left[-\frac{\pi}{2}, \frac{\pi}{2}\right]$ for each fixed $r>0$. It is not true that $\Re$ e $p\left(r e^{i \theta}\right)$ belongs to $L^{1}\left[-\frac{\pi}{2}, \frac{\pi}{2}\right]$, as can be seen by considering the functions $p_{r}(z)=(z-i r)^{-1}$. These functions show that the term $\cos \theta$ is necessary to cancel any possible (simple) poles on the imaginary axis.

The next result gives an interesting mapping property for positive functions $P$ which generalizes the result $(G)$ first proved by Brune [4] for positive real functions PR:

Theorem 2.9. If $p \in P$ and $p\left(z_{0}\right)>0$, where $z_{0}=x_{0}+i y_{0} \in H^{+}$, then

$$
\left|\operatorname{Arg}\left\{p\left(x_{0} e^{i \theta}+i y_{0}\right)\right\}\right| \leq|\theta|
$$

for $|\theta|<\frac{\pi}{2}$. This result is sharp.

Note that $(\mathrm{G})$ follows immediately from this theorem. Let $p \in P R$, fix $z=r e^{i \theta} \in$ $H^{+}$and choose $z_{0}=r$. Hence we have $p\left(z_{0}\right)>0$ and so

$$
|\operatorname{Arg} p(z)|=\left|\operatorname{Arg} p\left(r e^{i \theta}\right)\right| \leq|\theta|=|\operatorname{Arg} z| .
$$

Proof: Suppose $|\theta|<\frac{\pi}{2}$ is fixed. Consider the following extremal problem over $P(1)$ :

$$
\max _{q \in P(1)} \frac{\left|q\left(e^{i \theta}\right)\right|}{\Re e q\left(e^{i \theta}\right)} .
$$

Using Lemma 2.3 and Theorem 2.1(c) we may conclude that

$$
\frac{1}{\cos \left\{\operatorname{Arg} q\left(e^{i \theta}\right)\right\}}=\frac{\left|q\left(e^{i \theta}\right)\right|}{\Re e q\left(e^{i \theta}\right)} \leq \max _{q \in P(1)} \frac{\left|q\left(e^{i \theta}\right)\right|}{\Re e q\left(e^{i \theta}\right)}=\max _{q \in \mathcal{E}(P(1))} \frac{\left|q\left(e^{i \theta}\right)\right|}{\Re e q\left(e^{i \theta}\right)}=\frac{1}{\cos \theta}
$$

and hence

$$
\cos \left\{\operatorname{Arg} q\left(e^{i \theta}\right)\right\} \geq \cos \theta
$$

for all $q \in P(1)$. If $0 \leq \theta<\frac{\pi}{2}$, then the above inequality implies that $\left|\operatorname{Arg} q\left(e^{i \theta}\right)\right| \leq \theta$ (since $\left.\left|\operatorname{Arg} q\left(e^{i \theta}\right)\right|<\frac{\pi}{2}\right)$. If on the other hand $-\frac{\pi}{2}<\theta \leq 0$, then for any $q \in P(1)$ the function $f(z)=q\left(\frac{1}{z}\right) \in P(1)$ and hence

$$
\left|\operatorname{Arg} q\left(e^{i \theta}\right)\right|=\left|\operatorname{Arg} f\left(e^{-i \theta}\right)\right| \leq-\theta .
$$

In other words, for any $q \in P(1)$, we must have

$$
\left|\operatorname{Arg} q\left(e^{i \theta}\right)\right| \leq|\theta|
$$

Finally, apply (2.7) to the function $q(z)=p\left(x_{0} z+i y_{0}\right) / p\left(z_{0}\right)$ to obtain the result. Equality occurs for $p(z)=z-i y_{0}$.

Observe that Brune's result [4] implies that if $C$ is the circular arc $z=r e^{i \theta},|\theta| \leq$ $\mu<\pi / 2$, then the curve $p(C)$ lies in the sector $|\theta| \leq \mu$ for all positive real functions. 
The above theorem gives a similar result for the larger class of positive functions as follows. Let $z_{0}=x_{0}+i y_{0} \in H^{+}$and let $C_{0}$ be the circular arc (centered at $i y_{0}$ ) given by $z=i y_{0}+x_{0} e^{i \theta},|\theta| \leq \mu<\pi / 2$. If $p$ is any positive function satisfying $p\left(z_{0}\right)>0$, then $p\left(C_{0}\right)$ lies in the sector $|\theta| \leq \mu$.

\section{Positive Real Functions}

In the special case of positive real functions $P R$ we can improve the results of the previous section. For fixed $0<\alpha<\infty$, define $P R(\alpha)$ as the class of functions $p \in P R$ such that $p(1)=\alpha$. We first identify the extreme points for these classes.

Theorem 3.1. Let $0<\alpha<\infty$ be fixed.

(a) Each $p \in P R(\alpha)$ has a representation given by

$$
p(z)=\int_{-1}^{1} \frac{2 \alpha z}{\left(1+z^{2}\right)+t\left(1-z^{2}\right)} d \mu(t)
$$

where $\mu(t)$ is a probability measure on $[-1,1]$.

(b) $\operatorname{PR}(\alpha)$ is a compact convex family of functions.

(c) $\mathcal{E}(P R(\alpha))=\left\{\frac{2 \alpha z}{\left(1+z^{2}\right)+t\left(1-z^{2}\right)}:-1 \leq t \leq 1\right\}$.

Proof: Let $\mathbf{P}_{\mathbb{R}}$ denote the subclass of functions $q \in \mathbf{P}$ which satisfies $q(\bar{z})=\overline{q(z)}$ for $|z|<1$. Then it is known (see [5] or [8] for example) that each $q \in \mathbf{P}_{\mathbb{R}}$ has a representation

$$
q(z)=\int_{-1}^{1} \frac{1-z^{2}}{1-2 t z+z^{2}} d \mu(t)
$$

where $\mu(t)$ is a probability measure on $[-1,1]$. Moreover, the map $\mu \rightarrow q$ given by (3.1) is one-to-one and hence by [2, Theorem 1] the extreme points of the compact, convex family $\mathbf{P}_{\mathbb{R}}$ are given by

$$
\mathcal{E}\left(\mathbf{P}_{\mathbb{R}}\right)=\left\{\frac{1-z^{2}}{1-2 t z+z^{2}}:-1 \leq t \leq 1\right\} .
$$

Now let $p \in P R(\alpha)$ and observe that

$$
q(\zeta)=\frac{p\left(\frac{1+\zeta}{1-\zeta}\right)}{\alpha}=\int_{-1}^{1} \frac{1-\zeta^{2}}{1-2 t \zeta+\zeta^{2}} d \mu(t) .
$$

If we let $\zeta=(z-1) /(z+1)$, we obtain (a).

The statement (b) is now evident. The extreme points of $P R(\alpha)$ follow from (a) because the map $\mu \rightarrow q$ given in (3.1) is one-to-one and the mapping $\zeta=(z-1) /(z+1)$ is univalent. 
Clearly the bounds (F) on $|p(z)|$ given by Richards [12] and Seshu and Seshu [13] are not sharp. We are able to determine the precise sharp bounds.

Theorem 3.2. Let $p \in P R(\alpha)$ with $|\theta|<\frac{\pi}{2}$ and $r>0$.

(a) If $\cos 2 \theta \geq \min \left\{r^{2}, \frac{1}{r^{2}}\right\}$, then

$$
\alpha \min \left\{r, \frac{1}{r}\right\} \leq\left|p\left(r e^{i \theta}\right)\right| \leq \alpha \max \left\{r, \frac{1}{r}\right\} \text {. }
$$

(b) If $\cos 2 \theta \leq \min \left\{r^{2}, \frac{1}{r^{2}}\right\}$, then

$$
\alpha r\left|\frac{\sin 2 \theta}{1-r^{2} e^{2 i \theta}}\right| \leq\left|p\left(r e^{i \theta}\right)\right| \leq \frac{\alpha}{r}\left|\frac{1-r^{2} e^{2 i \theta}}{\sin 2 \theta}\right| .
$$

These results are all sharp.

Proof: For fixed $z=r e^{i \theta} \in H^{+}$, the functional $\left|p(z)\left(1+z^{2}\right) /(2 \alpha z)\right|$ is convex and hence by Lemma 2.2 and Theorem 3.1(c), we may conclude that for all $p \in P R(\alpha)$ :

$$
\left|\frac{p(z)\left(1+z^{2}\right)}{2 \alpha z}\right|^{2} \leq \max _{p \in \mathcal{E}(P R(\alpha))}\left|\frac{p(z)\left(1+z^{2}\right)}{2 \alpha z}\right|^{2}=\max _{-1 \leq t \leq 1} \frac{1}{h(t)}
$$

where $h(t)=|1+t w|^{2}=t^{2}|w|^{2}+2 t(\Re e w)+1$ and $w=\left(1-r^{2} e^{2 i \theta}\right) /\left(1+r^{2} e^{2 i \theta}\right)$.

Clearly the minimum of $h(t)$ occurs at $t_{0}=-\Re e w /|w|^{2}$, if $\left|t_{0}\right| \leq 1$. A check shows that $\left|t_{0}\right| \leq 1$ if and only if $\cos 2 \theta \leq r^{2}$ (for $0<r \leq 1$ ) or $\cos 2 \theta \leq r^{-2}$ (for $1 \leq r<\infty$ ). In other words, $\left|t_{0}\right| \leq 1$ if and only if $\cos 2 \theta \leq \min \left\{r^{2}, r^{-2}\right\}$.

(a): Assume that $\cos 2 \theta \geq \min \left\{r^{2}, r^{-2}\right\}$. It is evident that for all $-1 \leq t \leq 1$, if $\overline{0<} r \leq 1$ then $h(t) \geq h(-1)$; while if $r \geq 1$, then $h(t) \geq h(1)$. Using this and (3.2) we conclude that if $\cos 2 \theta \geq r^{2}$ (i.e., $\left.r \leq 1\right)$, then $\left|p\left(r e^{i \theta}\right)\right| \leq \alpha / r$ and if $\cos 2 \theta \geq r^{-2}$ (i.e., $r \geq 1$ ), then $\left|p\left(r e^{i \theta}\right)\right| \leq \alpha r$. These results then give

$$
\left|p\left(r e^{i \theta}\right)\right| \leq \alpha \max \left\{r, \frac{1}{r}\right\} .
$$

To obtain the lower bound, apply this inequality to $q(z)=\alpha^{2} / p(z)$. Equality is obtained only for the functions $p(z)=\alpha z$ and $p(z)=\alpha / z$.

(b): Assume that $\cos 2 \theta \leq \min \left\{r^{2}, r^{-2}\right\}$. By the observation made above, we see that the minimum of $h(t)$ occurs at $t_{0}$ and so

$$
h(t) \geq h\left(t_{0}\right)=\frac{|w|^{2}-(\Re e w)^{2}}{|w|^{2}}=\frac{2 r^{4}(1-\cos 4 \theta)}{\left|1-r^{4} e^{4 i \theta}\right|^{2}} .
$$

Using this and (3.2) we now deduce that

$$
\left|p\left(r e^{i \theta}\right)\right| \leq \frac{2 \alpha}{r} \frac{\left|1-r^{2} e^{2 i \theta}\right|}{\sqrt{2-2 \cos 4 \theta}}=\frac{\alpha}{r}\left|\frac{1-r^{2} e^{2 i \theta}}{\sin 2 \theta}\right| .
$$

The lower bound obtains from applying this inequality to $q(z)=\alpha^{2} / p(z)$. 
To see that these bounds are sharp, let $z_{0}=r e^{i \theta}$ be an arbitrary but fixed point in $H^{+}$and set $t=t_{0}$ in $p_{0}(z)=2 \alpha z /\left\{1+z^{2}+t\left(1-z^{2}\right)\right\}$, where $t_{0}=-\Re e w /|w|^{2}$ and $w=\left(1-r^{2} e^{2 i \theta}\right) /\left(1+r^{2} e^{2 i \theta}\right)$. Thus the functions $p_{0}(z)$ and $\alpha^{2} / p_{0}(z)$ give equality in (b). This completes the proof of the theorem.

Our theorem improves the result $(\mathrm{F})$ for all $z \in H^{+}$. Note that when $z=s>0$ is real, the bounds on $p(s)$ take a particularly simple form:

$$
\alpha \min \left\{\frac{1}{s}, s\right\} \leq p(s) \leq \alpha \max \left\{\frac{1}{s}, s\right\} .
$$

We can use these results to improve Theorem 2.6 for the class $P R(\alpha)$ for $\beta \neq 0$.

Theorem 3.3. Let $z=i \beta$, where $\beta \in \mathbb{R} \backslash\{0\}$, be an isolated singularity of $p \in P R(\alpha)$.

(a) If $z=i \beta$ is a zero of $p$, then $\left|p^{\prime}(i \beta)\right| \geq \frac{2 \alpha}{\beta^{2}+1}$. This is sharp.

(b) If $z=i \beta$ is a pole of $p$, then $|\operatorname{Res}\{p, i \beta\}| \leq \frac{\alpha}{2}\left(\beta^{2}+1\right)$. This is sharp.

Proof: We may assume without loss of generality that $\beta>0$.

Suppose first that $z=i \beta$ is a zero of $p \in P R(\alpha)$. Thus for some $\epsilon_{0}>0$ we have

$$
p(z)=\sum_{k=1}^{\infty} c_{k}(z-i \beta)^{k}
$$

valid for $|z-i \beta|<\epsilon_{0}$. Choose a fixed $\theta_{0}$ satisfying $0<\theta_{0}<\frac{\pi}{2}$ such that $\left|\beta e^{i \theta_{0}}-i \beta\right|<\epsilon_{0}$ and $\cos 2 \theta_{0} \leq \min \left\{\beta^{2}, \beta^{-2}\right\}$. Then for all $z=\beta e^{i \theta}$ with $\theta_{0}<\theta<\frac{\pi}{2}$, we may apply Theorem 3.2 to conclude that

$$
\begin{aligned}
\left|c_{1}+\sum_{k=2}^{\infty} c_{k}\left(\beta e^{i \theta}-i \beta\right)^{k-1}\right|^{2} & =\left|\frac{p\left(\beta e^{i \theta}\right)}{\beta e^{i \theta}-i \beta}\right|^{2} \\
& =\frac{\left|p\left(\beta e^{i \theta}\right)\right|^{2}}{2 \beta^{2}(1-\sin \theta)} \\
& \geq \frac{\alpha^{2}}{2\left|1-\beta^{2} e^{2 i \theta}\right|^{2}}\left(\frac{\sin ^{2} 2 \theta}{1-\sin \theta}\right) .
\end{aligned}
$$

This holds for all $\theta_{0}<\theta<\frac{\pi}{2}$. The conclusion (a) follows by letting $\theta \rightarrow \frac{\pi}{2}^{-}$. Equality holds for the function $p(z)=\frac{\alpha}{z}\left(\frac{z^{2}+\beta^{2}}{\beta^{2}+1}\right)$.

If $z=i \beta$ is a pole then we have

$$
p(z)=\sum_{k=1}^{N} \frac{b_{k}}{(z-i \beta)^{k}}+g(z),
$$


where $g(z)$ is analytic in $|z-i \beta|<\epsilon_{0}$ for some $\epsilon_{0}>0$. As above we choose $\theta_{0}$ so that $\left|\beta e^{i \theta_{0}}-i \beta\right|<\epsilon_{0}$ and $\cos 2 \theta_{0} \leq \min \left\{\beta^{2}, \beta^{-2}\right\}$. For all $z=\beta e^{i \theta}$ and $\theta_{0}<\theta<\frac{\pi}{2}$, use Theorem 3.2 to obtain

$$
\begin{aligned}
\left|b_{1}+\sum_{k=2}^{N} \frac{b_{k}}{\left(\beta e^{i \theta}-i \beta\right)^{k-1}}+\left(\beta e^{i \theta}-i \beta\right) g\left(\beta e^{i \theta}\right)\right| & =\beta \sqrt{2-2 \sin \theta}\left|p\left(\beta e^{i \theta}\right)\right| \\
& \leq \alpha\left|1-\beta^{2} e^{2 i \theta}\right| \frac{\sqrt{2-2 \sin \theta}}{\sin 2 \theta} .
\end{aligned}
$$

If we let $\theta \rightarrow \frac{\pi}{2}^{-}$, then it follows that $b_{k}=0$ for $k=2,3, \cdots, N$ and $\left|b_{1}\right| \leq \frac{\alpha}{2}\left(\beta^{2}+1\right)$. Equality occurs for $p(z)=\frac{\alpha z\left(\beta^{2}+1\right)}{z^{2}+\beta^{2}}$. This completes the proof of the theorem.

If $\beta=0$, then Theorem 2.6 already yields sharp estimates on $\Re e p^{\prime}(0)$ and $|\operatorname{Res}\{p, 0\}|$ for $p \in P R(\alpha)$, with equality for $p(z)=\alpha z$ and $p(z)=\alpha / z$, respectively.

The next two results essentially state that the resistance and reactance terms of the complex impedance are comparable to $p(r)$ in a sector:

Theorem 3.4. If $p \in P R(\alpha)$, then

$$
p(r) \cos \theta \leq \Re e p\left(r e^{i \theta}\right) \leq\left|p\left(r e^{i \theta}\right)\right| \leq \frac{p(r)}{\cos \theta}
$$

for $|\theta|<\frac{\pi}{2}$ and $r>0$. This result is sharp.

Proof: Let us suppose first that $z=e^{i \theta}$ is fixed. Then by Theorem 3.1(c) and Lemma 2.2 we can conclude that for each $q \in P R(\alpha)$

$$
\min _{-1 \leq t \leq 1} \Re e Q\left(e^{i \theta}\right) \leq \Re e\left\{\frac{q\left(e^{i \theta}\right)}{2 \alpha}\right\} \leq \max _{-1 \leq t \leq 1} \Re e Q\left(e^{i \theta}\right),
$$

where $Q(z)=\frac{z}{\left(1+z^{2}\right)+t\left(1-z^{2}\right)}$. A calculation shows that

$$
\Re e Q\left(e^{i \theta}\right)=\frac{\cos \theta}{2 \cos ^{2} \theta+2 t^{2} \sin ^{2} \theta}
$$

which gives

and so

$$
\frac{\cos \theta}{2} \leq \Re e Q\left(e^{i \theta}\right) \leq \frac{1}{2 \cos \theta}
$$

$$
\alpha \cos \theta \leq \Re e q\left(e^{i \theta}\right) \leq \frac{\alpha}{\cos \theta} .
$$

Now for fixed $r>0$, let $q(z)=\alpha p(r z) / p(r)$ and apply inequality (3.3) with $z=e^{i \theta}$ to obtain

$$
p(r) \cos \theta \leq \Re e p\left(r e^{i \theta}\right) \leq \frac{p(r)}{\cos \theta} .
$$


This also gives $p(r) \cos \theta \leq\left|p\left(r e^{i \theta}\right)\right|$. Apply this inequality to $q(z)=\alpha p(r) / p(r z)$ to obtain the upper bound for $\left|p\left(r e^{i \theta}\right)\right|$.

For fixed $r>0$, the lower bound is achieved for $p(z)=\alpha z$ or $p(z)=\alpha / z$, while the upper bound is achieved for $p(z)=\alpha z\left(\frac{1+r^{2}}{z^{2}+r^{2}}\right)$.

There seems to be no information in the literature concerning the reactance part of positive real functions, i.e., $\Im m p(z)$. The following result appears to be the first of its kind.

Theorem 3.5. If $p \in P R(\alpha)$, then

This result is sharp.

$$
\left|\Im m p\left(r e^{i \theta}\right)\right| \leq\left\{\begin{array}{cl}
p(r)|\sin \theta|, & 0 \leq|\theta| \leq \frac{\pi}{4} \\
\frac{p(r)}{2 \cos \theta}, & \frac{\pi}{4} \leq|\theta|<\frac{\pi}{2}
\end{array} .\right.
$$

Proof: Suppose first that $z=e^{i \theta}$ is fixed. For $q \in P R(1)$, the functional $\left|\Im m q\left(e^{i \theta}\right)\right|$ is convex and so by Lemma 2.2 and Theorem 3.1(c) we conclude that

$$
\left|\Im m q\left(e^{i \theta}\right)\right| \leq \max _{-1 \leq t \leq 1} \Psi(t) .
$$

where $\Psi(t)=\frac{|t \sin \theta|}{\cos ^{2} \theta+t^{2} \sin ^{2} \theta}$. A brief calculation shows that if $0 \leq|\theta| \leq \frac{\pi}{4}$, then $\Psi(t) \leq \Psi(1)$; while if $\frac{\pi}{4} \leq|\theta|<\frac{\pi}{2}$, then $\Psi(t) \leq \Psi(|\cot \theta|)$. To complete the proof, we apply these inequalities to the function $q(z)=p(r z) / p(r)$.

To demonstrate sharpness, without loss of generality, we may assume $0 \leq \theta<\frac{\pi}{2}$ and is fixed. We define $q_{1}(z)=\alpha z$ and $q_{2}(z)=2 \alpha z /\left(1+z^{2}+\cot \theta\left(1-z^{2}\right)\right)$. For fixed $r>0$, we then see that the functions $p_{k}(z)=\alpha \frac{q_{k}\left(\frac{z}{r}\right)}{q_{k}\left(\frac{1}{r}\right)}$ for $k=1,2$ give equality for $0 \leq \theta \leq \frac{\pi}{4}$ and $\frac{\pi}{4} \leq \theta<\frac{\pi}{2}$, respectively.

We point out that the result $(\mathrm{G})$ of Brune [4] says for example, the image of the sector $|\arg z| \leq \phi<\frac{\pi}{2}$ under a positive real function $w=p(z)$ lies in the sector $|\arg w| \leq \phi$. If $p(r)$ is bounded our result says that the image actually lies in a horizontal strip which depends only on the bound for $p(r)$ and $\phi$.

Note also that we obtain a Tsuji-type result (E) for $\Im m p(z)$ for $p \in P R(\alpha)$ whose proof follows immediately from the above theorem:

Corollary 3.6. If $p \in P R(\alpha)$ and $p(r) \leq M$, for $0<r \leq 1$, then $|\Im m p(z)|$ is uniformly bounded in the sector $|\arg z| \leq \phi,|z| \leq 1$ for any $0 \leq \phi<\frac{\pi}{2}$. 
Since the extremal functions for the upper bounds on $\left|p^{(n)}(z)\right|$ given in (D) are not real the question of determining these sharp upper bounds for functions in $P R$ has been open since 1932. Suppose we fix $|\theta|<\pi / 2$ and let $\alpha=1$. Then we may apply Lemma 2.3 and Theorem 2.1(c) to the quotient $\left|q^{\prime}\left(e^{i \theta}\right)\right| / \Re e\left\{q\left(e^{i \theta}\right)\right\}$ over the class $P R(1)$ to obtain

$$
\frac{\left|q^{\prime}\left(e^{i \theta}\right)\right|}{\Re e\left\{q\left(e^{i \theta}\right)\right\}} \leq \max _{q \in \mathcal{E}(P(1))} \frac{\left|q^{\prime}\left(e^{i \theta}\right)\right|}{\Re e\left\{q\left(e^{i \theta}\right)\right\}}=\max _{-1 \leq t \leq 1} \frac{|-i \sin \theta+t \cos \theta|}{\cos \theta} \leq \frac{1}{\cos \theta},
$$

with equality only if $t= \pm 1$, i.e., only if $q(z)=z$ and $q(z)=1 / z$. Now let $r>0$ be fixed and let $p$ be an arbitrary function in $P R(\alpha)$. Apply the above result to $q(z)=p(r z) / p(r)$ to obtain $\left|p^{\prime}\left(r e^{i \theta}\right)\right| \leq \Re e\left\{p\left(r e^{i \theta}\right)\right\} / \Re e\left\{r e^{i \theta}\right\}$, with equality only for $p(r z) / p(r)=z$ or $1 / z$. These give $p(z)=\alpha z$ or $p(z)=\alpha / z$. Hence we have proved:

Theorem 3.7. If $p \in P R(\alpha)$ and $z \in H^{+}$, then

$$
\left|p^{\prime}(z)\right| \leq \frac{\Re e\{p(z)\}}{\Re e\{z\}} .
$$

Equality is attained only for $p(z)=\alpha z$ and $p(z)=\frac{\alpha}{z}$.

This gives an alternate proof of (D) for $P R$ and $n=1$ and, in addition, shows that one can do no better than (D) for $n=1$ when restricting to positive real functions.

Early attempts at estimating $\left|p^{\prime}(z)\right|$ in terms of $p(1)$ had been made. Richards [12] using his estimates in (F) showed that $\left|p^{\prime}(z)\right|<p(1)\left(|z|^{2}+1\right) / \Re e\{z\}$. Also it should be pointed out that for the special case $z=s>0$, Seshu and Seshu [13] proved that

$$
\left|p^{\prime}(s)\right|<p(1)\left(4 s^{2}+2\right) / s^{2} .
$$

By combining our above theorem with Theorem 3.2(a), we obtain the sharp estimate

$$
\left|p^{\prime}(s)\right| \leq p(1) \max \left\{1, \frac{1}{s^{2}}\right\}
$$

which vastly improves (3.4) for all $s>0$.

The case of $\left|p^{\prime \prime}(z)\right|$ is quite different and much more involved. Here the estimate for (D) when $n=2$ can be improved for the class $P R$. For convenience, we define the functions $\phi(t, x)$ and $\Phi(x)$ as follows:

$$
\phi(t, x)=\frac{(1-x)(1-t)^{2}\left[(2 t+1)^{2}+3 x\left(1-t^{2}\right)\right]}{1-x+x t^{2}}
$$

and

$$
\Phi(x)=\max _{-1 \leq t \leq 1} \sqrt{\frac{(1-x)}{4} \phi(t, x)} .
$$

We can now state the result. 
Theorem 3.8. If $p \in P R(\alpha)$ and $z=r e^{i \theta} \in H^{+}$, then

$$
\left|p^{\prime \prime}(z)\right| \leq \frac{2 ! \Re e\{p(z)\}}{(\Re e\{z\})^{2}} \Phi\left(\sin ^{2} \theta\right) .
$$

This result is sharp for each fixed $z=r e^{i \theta} \in H^{+}$.

Remark: It is not difficult to show that $(1-x) \phi(t, x) \leq 4$ with equality only for $t=-1$ and $x=0$. Hence $\Phi(x) \leq 1$ and $p(z)=\alpha / z$ is thus extremal only when $z$ is real. This also gives another proof of (D) for $n=2$.

Proof: Fix $|\theta|<\pi / 2$ and let $\alpha=1$. As above, we consider this time the functional

$$
\frac{\left|q^{\prime \prime}\left(e^{i \theta}\right)\right|}{\Re e\left\{q\left(e^{i \theta}\right)\right\}} \text {. }
$$

Apply Lemma 2.3 and Theorem 2.1(c) and let $x=\sin ^{2} \theta$ to conclude, after a long but straightforward calculation, that if $q \in P R(1)$ then

$$
\frac{\left|q^{\prime \prime}\left(e^{i \theta}\right)\right|}{\Re e\left\{q\left(e^{i \theta}\right)\right\}} \leq \max _{q \in \mathcal{E}(P(1))} \frac{\left|q^{\prime \prime}\left(e^{i \theta}\right)\right|}{\Re e\left\{q\left(e^{i \theta}\right)\right\}}=\max _{-1 \leq t \leq 1} \sqrt{\frac{\phi(t, x)}{1-x}}=\frac{2 \Phi(x)}{1-x} .
$$

The last equality follows from (3.6). Thus we conclude that if $q \in P R(1)$, then

$$
\frac{\left|q^{\prime \prime}\left(e^{i \theta}\right)\right|}{\Re e\left\{q\left(e^{i \theta}\right)\right\}} \leq \frac{2 \Phi\left(\sin ^{2} \theta\right)}{\cos ^{2} \theta}
$$

For fixed $|\theta| \frac{\pi}{2}$, equality occurs only for $q_{k}(z)=\frac{2 z}{\left(1+z^{2}\right)+t_{k}\left(1-z^{2}\right)}$, where $t_{k} \in$ $[-1,1]$ with $\phi\left(t_{k}, \sin ^{2} \theta\right)=\max _{-1 \leq t \leq 1} \phi\left(t, \sin ^{2} \theta\right)$. Fix $r>0$ and apply inequality (3.7) to $q(z)=p(r z) / p(r)$ to obtain the desired inequality.

To demonstrate sharpness, fix $r>0$ and $|\theta|<\pi / 2$. By the above, equality is attained for $\frac{p(r z)}{p(r)}=q_{k}(z)=\frac{2 z}{\left(1+z^{2}\right)+t_{k}\left(1-z^{2}\right)}$. A calculation then gives

$$
p(z)=\alpha z\left[\frac{\left(r^{2}+1\right)+t_{k}\left(r^{2}-1\right)}{\left(r^{2}+z^{2}\right)+t_{k}\left(r^{2}-z^{2}\right)}\right] .
$$

Estimates on $\left|p^{\prime \prime}(z)\right|$ were given by Pearson [10]. In fact, he rediscovered the result (D) of Wolff and DeKoK [16] in the special case $n=2$. We remark that we can also use the same techniques as above to determine sharp bounds on $\left|p^{(n)}(z)\right|$ for any $n>1$, but the calculations are tedious. 


\section{REMARKS}

(1) Brune [4] proved that to every positive real function with a finite number of zeros and poles in $\mathbb{C} \backslash H^{+}$, there corresponds a finite physically realizable electric network. It is important to note that all our extremal functions are precisely of this form.

(2) Linear fractional transformations are an important tool in the study of positive functions. For example, a simple observation gives this result of Brune [4]: if $p \in P R$ then the equation $p(\lambda)=L \lambda$, where $L>0$, cannot have more than one solution in the right half-plane. This follows immediately by using suitable linear fractional transformations. Indeed, if $p \in P R$ (and $p(z) \neq \alpha z$ or $\alpha z^{-1}$ ), then it follows that for any $\mu>0$ the function $q(z)=\frac{\mu p(z)-z p(\mu)}{\mu p(\mu)-z p(z)}$, the Richards' transformation, is also in $P R$ (see [12] or [6]). From this we can conclude that

$$
\mu p(z)-z p(\mu) \neq 0 \text { for } \Re e z>0 \text { and } z \neq \mu \text {. }
$$

Assume say $p(\lambda)=L \lambda$ for some $\lambda>0$, then for $\mu=\lambda$, the expression (4.1) implies that $p(z) \neq L z$, for all $z \neq \lambda$. Another interesting fact to point out is that since $q(\mu)=\left(p(\mu)-\mu p^{\prime}(\mu)\right) /\left(p(\mu)+\mu p^{\prime}(\mu)\right)$, it follows that $p^{\prime}(s) \neq \frac{p(s)}{s}$ (or $-p(s) / s$ ) for all $s>0$ and all $p \in P R$ unless $p(z)=\alpha z$ (or $\left.\alpha z^{-1}\right)$. This is a rather curious fact in view of the result $(\mathrm{A})$.

(3) Finally, there is a connection between $P$ and the classical family $S$ (those functions $f$ analytic and univalent in $|z|<1$ and normalized so that $f(z)=$ $\left.z+a_{2} z^{2}+\cdots\right)$. For any $p \in P\left(\omega_{0}\right)$, the function $g(\zeta)=\int_{1}^{\zeta} p(\omega) d \omega$ is analytic in the convex region $\Re e \zeta>0$. By the Noshiro-Warshawski Theorem (see Duren [5], page 47), the function $g(\zeta)$ is univalent. After a suitable linear fractional transformation and a normalization we see that $f(z)=g((1+$ $z) /(1-z)) /\left(2 \omega_{0}\right) \in S$. From this we obtain

$$
p\left(\frac{1+z}{1-z}\right)=\omega_{0}(1-z)^{2} f^{\prime}(z),|z|<1
$$

where $f \in S$. Using (4.2) and for example the well-known result that $\left|a_{2}\right| \leq 2$, the inequality

$$
\left|\frac{p^{\prime}(1)}{\omega_{0}}+1\right| \leq 2
$$

obtains. Other results follow from (4.2) and properties of functions in $S$. 


\section{REFERENCES}

[1] R. Bott and R. Duffin, Impedance Synthesis Without the Use of Transformers, J. Appl. Phys. 20(1949), 816.

[2] L. Brickman, T. H. MacGregor and D. R. Wilken, Convex Hulls of Some Classical Families of Univalent Functions, Trans. Amer. Math. Soc. 156(1971), 91-107.

[3] J. E. Brown, Applications of Extreme Points to Distortion Estimates, Complex Variables Theory and Appl. 47(2002), 229-237.

[4] O. Brune, Synthesis of a Finite Two-Terminal Network Whose Driving Point Impendance is a Prescribed Function of Frequency, J. of Math. and Physics 10 (1931), 191-236 .

[5] P. L. Duren (1983), Univalent Functions, Springer-Verlag, Berlin and New York.

[6] J. L. Goldberg, Functions With Positive Real Part In a Half-Plane, Duke Math. J. 29(1962), 333-340.

[7] J. L. Goldberg, Bounds On the Derivative of Positive Functions, SIAM Rev. 8(1966), 343-345.

[8] D. J. Hallenbeck and T. H. MacGregor (1984), Linear Problems and Convexity Techniques in Geometric Function Theory, Monographs and Studies in Mathematics 22, Pitman.

[9] E. Landau and G. Valiron, A Deduction From Schwarz's Lemma, J. London Math. Soc. 4(1929), 162-163.

[10] C. Pearson, A Note On the Second Derivative of a P.R. Function, SIAM Rev 7(1965), 251-252.

[11] F. Reza, A Bound for the Derivative of a Positive Real Function, SIAM Rev. 4(1962), 40-42.

[12] P. I. Richards, A Special Class of Functions With Positive Real Part In a Half-Plane, Duke Math. J. 14(1947), 777-786.

[13] S. Seshu and L. Seshu, Bounds and Stieltjes Transform Representations for Positive Real Functions, J. Math. Anal. Appl. 3(1961), 592-604.

[14] M. Tsuji, On a Positive Harmonic Function In a Half Plane, J. Math. Soc. Japan 7(1955), 76-78.

[15] M. Tsuji (1959), Potential Theory in Modern Function Theory, Tokyo.

[16] J. Wolff and F. DeKok, Les Fonctions Holomorphes á Partie Réelle Positive et L'intégral de Stieltjes, Bull. de la Soc. Math. de France 60(1932), 221-227.

Current address: Department of Mathematics, Purdue University, West Lafayette, IN 47907 